\title{
HANSENÍASE EM POVOS INDÍGENAS DO MARANHÃO NO PERÍODO DE 2016 A 2020
}

\author{
LEPROSY IN INDIGENOUS PEOPLES OF MARANHÃO IN THE PERIOD FROM 2016 \\ TO 2020
}

\author{
Julia Pinheiro Martins ${ }^{1}$ \\ Thainá Furtado Praxedes ${ }^{2}$ \\ Ilaila Coelho Guajajara ${ }^{3}$ \\ Geórgia Marreiros de Sousa ${ }^{4}$
}

RESUMO: O artigo tem por objetivo, descrever os aspectos históricos e epidemiológicos da hanseníase dentro da população indígena. A hanseníase é uma doença infectocontagiosa, causada pelo Mycobacterium leprae, historicamente conhecida como lepra, é considerada uma das doenças mais antigas do mundo. É transmitida através do contato com pacientes multibacilares e pode se manifestar através de sinais e sintomas dermatológicos e neurológicos como lesões na pele e nos nervos periféricos, principalmente na região dos olhos, mãos e pés. Este estudo objetivou identificar os casos de hanseníase por lesões cutâneas notificados no Estado do Maranhão nos períodos de 2016 a 2020. De natureza descritiva e quantitativa, a metodologia utilizada foi a pesquisa na base de dados no Sistema de Informação de Agravos de Notificação (SINAN) sobre as ocorrências de hanseníase na população indígena notificadas no estado do Maranhão, delineando seu perfil epidemiológico. Os resultados obtidos no estudo, revelaram que no período de 2016/20, foram registrados 46I casos de hanseníase em indígenas, desse montante a maioria é do sexo masculino, faixa etária entre I5-19 anos, forma clínica e o ano de maior prevalência dos casos. À vista dos dados, ressalta-se a importância da Vigilância Epidemiológica do Estado e dos Municípios, das notificações e dos trabalhos educativos junto a população indígena.

Palavras-chaves: Hanseníase. Mycobacterium leprae. População indígena.

ABSTRACT: The article aims to describe the historical and epidemiological aspects of leprosy within the indigenous population. Leprosy is an infectious disease caused by Mycobacterium leprae, historically known as leprosy, and is considered one of the oldest diseases in the world. It is transmitted through contact with multibacillary patients and can manifest itself through dermatological and neurological signs and symptoms such as lesions on the skin and peripheral nerves, especially in the region of the eyes, hands, and feet. This study aimed to identify the cases of leprosy by skin lesions reported in the State of Maranhão in the periods from 2016 to 2020. Descriptive and quantitative in nature, the methodology used was the database search in the Information System of Notifiable Diseases (SINAN) on the occurrences of leprosy in the indigenous population notified in

\footnotetext{
${ }^{1}$ Acadêmica de Enfermagem pela Universidade Estadual do Maranhão-UEMA. E-mail: julia.pinheiro.214@gmail.com.

${ }^{2}$ Acadêmica de Enfermagem pela Universidade Estadual do Maranhão-UEMA. E-mail: thainapraxedes34@gmail.com.

3 Acadêmica de Enfermagem pela Universidade Estadual do Maranhão-UEMA E-mail: lailacoelho92@gmail.com.

${ }_{4}^{4}$ Acadêmica de Enfermagem pela Universidade Estadual do Maranhão-UEMA E-mail: benjamingjmk@gmail.com.
} 
the state of Maranhão, outlining its epidemiological profile. The results obtained in the study, revealed that in the period 2016/20, 46I cases of leprosy in indigenous people were registered, of this amount the majority is male, age group between $15^{-19}$ years, clinical form and the year of higher prevalence of cases. In view of the data, the importance of the Epidemiological Surveillance of the State and Municipalities, the notifications and the educational work with the indigenous population should be emphasized.

ABSTRACT: Leprosy. Mycobacterium leprae. Indigenous population.

\section{INTRODUÇÃO}

A hanseníase, historicamente conhecida como lepra, é considerada uma das doenças mais antigas do mundo, consta-se que a mesma tenha surgido entre exploradores, comerciantes e colonizadores de origem europeia ou do norte africano. No Brasil surge juntamente com a colonização do país, os povos indígenas que habitavam no litoral estavam constantemente próximos aos primeiros colonizadores portugueses e consequentemente iam sendo infectados (TERRA,I995; MARTINS,2009).

No início do século $\mathrm{XX}$, a hanseníase tornou-se um grande problema de saúde pública, passando então a ser controlada pelo Estado. Medidas de internamento compulsório tornaram-se presentes, em locais que eram conhecidos como "leprosários", com o objetivo de conter a disseminação da doença, no entanto, essas pessoas eram segregadas da sociedade, e mesmo com a descoberta dos medicamentos para tratamento e cura e, com isso, o fim do isolamento, o estigma e o preconceito permanecem enraizados na sociedade. Cícero Fraga de Melo (2017) aponta que o estigma não foi instituído pelo medo de contrair a doença, mas pelos sinais extremamente visíveis no corpo e isso resultava no sofrimento, retardo da descoberta e tratamento.

Trata-se de uma doença crônica, infectocontagiosa, cujo agente etiológico é o Mycobacterium leprae, um bacilo que infecta os nervos periféricos, e mais especificamente as células de Schwann. A doença acomete, principalmente, os nervos superficiais da pele e os troncos nervosos periféricos e pode afetar os olhos e os órgãos internos (BRASIL, 2017).

A definição de caso de hanseníase, de acordo com a Organização Mundial da Saúde (OMS), baseia-se em apresentar uma ou mais das seguintes características: Pelo menos uma lesão de pele com alteração da sensibilidade; acometimento de nervo com espessamento neural ou, baciloscopia positiva para M. leprae (AZULAY, 2017). O diagnóstico é essencialmente clínico, realizado através de anamnese, exame geral e dermatoneurológico, 
que objetiva identificar áreas de pele que apresentem alterações sensitivas, motoras ou autonômicas. Pode ser classificada em Paucibacilar (PB) - casos com até cinco lesões de pele ou Multibacilar (MB)- casos com mais de cinco lesões de pele. O tratamento é realizado com a associação de medicamentos (poliquimioterapia- PQT) como rifampicina, dapsona e clofazimina, deve ser iniciado já na primeira consulta, depois que o diagnóstico é definido (BRASIL, 2017)

De acordo com Ivanir Ferreira (2020), o Brasil ocupa a segunda posição ao se tratar de novos casos de hanseníase e carrega 92\% das ocorrências das Américas. Essa intercorrência soma-se às questões econômicas, sociais e ambientais, que se mostram presentes no processo de adoecimento das populações.

Com 305 povos e 274 línguas diferentes, a população indígena brasileira vive realidades diversas que envolvem desde grupos isolados até os que residem em áreas urbanas, apresentando grande diversidade histórica, social e cultural, a socialização destes povos com povos não indígenas, vem gerando inúmeras doenças infectocontagiosas, dentre elas a hanseníase, devido à condições de moradia, baixas condições socioeconômicas e serviços de saúde inadequados (SILVA,20II)

Outro problema decorrente na saúde dos povos indígenas, trata-se do sistema de informação em saúde que ainda é precário devido à carência na alimentação do mesmo pelos profissionais habilitados para isto, fato pelo qual pode se explicar as subnotificações e subdiagnósticos (CARVALHO,2012).

Este estudo objetivou identificar os casos de hanseníase por lesões cutâneas notificados no Estado do Maranhão nos períodos de 2016 a 2020.

\section{METODOLOGIA}

Trata-se de um estudo descritivo e quantitativo, e se deu através de uma pesquisa bibliográfica, abordando a hanseníase na população indígena, o delineamento do perfil epidemiológico deu-se pelo levantamento do número de casos notificados no Estado do Maranhão nos meses de agosto de 202I à setembro de 202I, referente ao intervalo dos anos de 2016 a 2020 . 
A consolidação dos dados foi realizada através do Sistema de Informação de Agravos de Notificação (SINAN) e da base de dados disponibilizada pelo Departamento de Informática do Sistema Único de Saúde (DATASUS).

No que se refere a caracterização do estado, o mesmo inclui-se na macrorregião do nordeste brasileiro, limitado pelo Oceano Atlântico e pelos estados Piauí, Tocantins e Pará. No seu território existem 217 municípios, com 16 terras indígenas (TI), distribuídas em cinco mesorregiões, sendo norte, sul, leste, oeste e centro maranhense. Possui área territorial estimada em $329.651 .495 \mathrm{~km}^{2}$, densidade demográfica em $19,8 \mathrm{r}$ hab $/ \mathrm{km}^{2}$ e população indígena estimada em 35.272 habitantes e pelo menos 25 mil têm residência nas comunidades indígenas. O Maranhão ocupa a $9^{\underline{a}}$ posição no ranking de maior população indígena do país, representando 4,3\% da população no país (IBGE, 20I0).

Para a realização desta pesquisa, foram realizadas buscas eletrônicas de artigos em periódicos brasileiros, a busca por produções científicas incluiu pesquisas nas bases de dados, PubMed, Scientific Electronic Library Online (SciELO) e Google Acadêmico.

Como critério de inclusão, considerou-se artigos e artigos de revisão teórica publicados no idioma português. Como critérios de exclusão foram considerados artigos que não estivessem no idioma português e que não atendiam aos objetivos propostos.

Foram consideradas como variáveis, os casos confirmados por lesões cutâneas, sexo, faixa etária, ano de notificação, classificação operacional, forma clínica e esquema terapêutico.

\section{RESULTADOS E DISCUSSÃO}

Segundo os dados do SINAN (Sistema de Informação de Agravamentos de Notificação), foram registrados 46r casos de hanseníase no Estado do Maranhão no período em análise. Observou-se que o ano de maior prevalência foi o de 20ı6, foram notificados ao todo 169 casos ( $\approx 3,66 \%$ maior em relação aos demais anos).

As características sociodemográficas da população indígena foram resumidas no Gráfico, no qual pode se notar a alta prevalência da doença por lesões cutâneas em pessoas do sexo masculino $(64,2 \%)$ e no sexo feminino $(35,7 \%)$. 
Gráfico I. Casos confirmados e notificados de hanseníase por lesões cutâneas, conforme sexo no período de 2016 a 2020

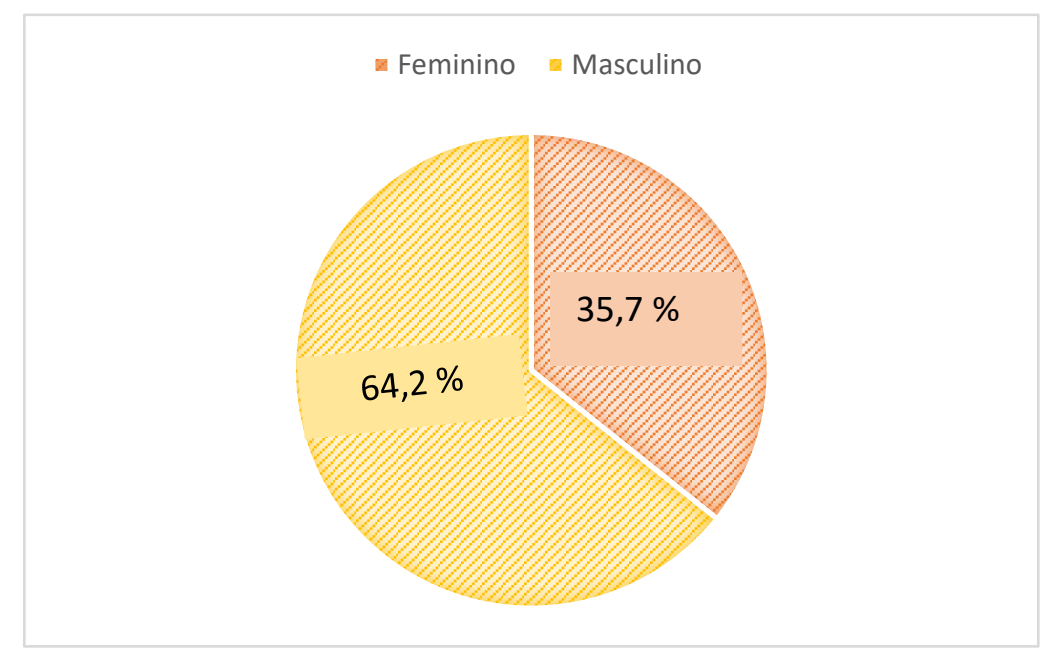

Fonte: Ministério da Saúde/ SVS- Sistema de Informação de Agravos de Notificação- Sinan Net, 202I.

A Tabela I apresenta os casos de hanseníase em indígenas segundo faixa etária, pode ser observado que a quantidade de casos de hanseníase foi entre os indivíduos que apresentam de is a I9 anos. O Ministério da Saúde afirma que a hanseníase pode atingir todas as idades e ambos os sexos, no entanto os menores de 15 anos são considerados indicador de alta endemicidade da doença, e há uma incidência maior da doença nos homens do que nas mulheres. Saito \& Silva afirmam que "a adolescência é uma etapa fundamental para a construção do ser humano, e, é resultante de tudo que a precedeu e determinante de tudo o que há de vir”. A hanseníase é uma doença estigmatizante, que acarreta transtornos físicos e psicológicos, para um adolescente que está numa fase de mudanças e de adaptações à hanseníase pode interferir na construção de sua vida.

Tabela r. Lesões cutâneas por forma clínica.

\begin{tabular}{|l|l|}
\hline Faixa etária & Total \\
\hline I -4 & - \\
\hline $5-9$ & 14 \\
\hline I0 - I4 & 8 \\
\hline I5 - I9 & II2 \\
\hline $20-29$ & 28 \\
\hline $30-39$ & 77 \\
\hline $40-49$ & 5 \\
\hline
\end{tabular}




\begin{tabular}{|l|l|}
\hline $50-59$ & 74 \\
\hline $60-69$ & 66 \\
\hline $70-79$ & 52 \\
\hline 80 ou + & 25 \\
\hline Total & 46 I \\
\hline
\end{tabular}

Fonte: Ministério da Saúde/ SVS- Sistema de Informação de Agravos de Notificação- Sinan Net, 2021.

Quanto às formas clínicas (Tabela 2), a dimorfa apresentou maior prevalência, totalizando 185 casos notificados. As formas clínicas dimorfa e virchowiana, são as mais infectantes, sua transmissão é facilitada através das vias aéreas por onde pode ocorrer a eliminação dos bacilos (LIMEIRA OM, et al., 2013). A hanseníase dimorfa é a forma que apresenta maior variedade de lesões, que podem se manifestar como placas, nódulos eritematosos e acastanhados, geralmente mais numerosas e tendendo à simetria. Acometimento mais extenso de nervos, que podem evoluir com neurites agudas graves (ARAÚJO, 2003). Outro estudo realizado na população indígena mostrou resultados semelhantes relacionados à forma clínica, constatou-se que a forma dimorfa foi a mais frequente (TEOFILO, 2014).

Tabela 2. Lesões cutâneas por forma clínica.

\begin{tabular}{|l|l|}
\hline Variáveis & Total \\
\hline Ign branco & I0 \\
\hline Tuberculóide & $9 \mathrm{I}$ \\
\hline Indeterminada & 28 \\
\hline Dimorfa & I85 \\
\hline Virchowiana & 140 \\
\hline Não classificada & 7 \\
\hline Total & $46 \mathbf{I}$ \\
\hline
\end{tabular}

Fonte: Ministério da Saúde/ SVS- Sistema de Informação de Agravos de Notificação- Sinan Net, 2021.

No que se refere à classificação operacional, podemos observar na Tabela 3 a distribuição do número de casos da hanseníase em indígenas, 48 casos notificados são referentes à classificação operacional Paucibacilar e 413 Multibacilar. Observou-se ainda, que a classificação operacional multibacilar foi mais presente no sexo masculino, isto pode 
ser explicado pelo fato dos homens, geralmente, procurarem o serviço de saúde já no estágio mais avançado da doença (SANTOS, 2012).

Tabela 3. Lesões cutâneas por sexo, segundo classificação operacional.

\begin{tabular}{|l|c|c|c|}
\hline Sexo & Paucibacilar (PB) & Multibacilar (MB) & Total \\
\hline Masculino & $4 \mathrm{I}$ & 255 & 296 \\
\hline Feminino & 7 & 158 & 165 \\
\hline
\end{tabular}

Fonte: Ministério da Saúde/ SVS- Sistema de Informação de Agravos de NotificaçãoSinan Net, 2021.

$\mathrm{Na}$ Tabela 4 é apresentada a forma de tratamento a que os indígenas com hanseníase foram submetidos, I4 casos receberam o esquema terapêutico PQT/PB/6 doses e 447 $\mathrm{PQT} / \mathrm{MB} / \mathrm{r2}$. O tratamento do paciente com hanseníase é iniciado assim que ele é classificado, é determinado qual será o esquema terapêutico. A hanseníase tem cura através do uso da PQT, além do benefício da cura, a transmissão da doença é interrompida (GUSSO, 2018).

O tratamento completo é importante na medida em que evita o surgimento das complicações da hanseníase, as quais são motivos de segregação dos pacientes, seja pelas incapacidades físicas associadas a elas, que dificultam a qualidade de vida, bem como a manutenção no mercado de trabalho, seja pela discriminação social propriamente dita devido à estranheza causada pelas manifestações clínicas da doença (BRASIL, 20I9)

Tabela 4. Esquema Terapêutico 2016-2020.

\begin{tabular}{|c|c|c|c|}
\hline Esquema terapêutico & Masculino & Feminino & Total \\
\hline PQT/PB/6 DOSES & 7 & 7 & 14 \\
\hline PQT/MB/12 DOSES & 289 & 158 & 447 \\
\hline Total & 296 & 165 & 46 I \\
\hline
\end{tabular}

Fonte: Ministério da Saúde/ SVS- Sistema de Informação de Agravos de Notificação- Sinan Net, 2021. 
No período estudado, dos 46I casos notificados pelo SINAN, 286 tiveram encerramento devido à alta por cura e foram observados 34 casos de abandono, somente por homens. $\mathrm{O}$ abandono do tratamento refere-se ao paciente que não completou o total de doses de medicação no prazo estimado, ou aquele que não foi registrado o comparecimento nas unidades de saúde no período de um ano (BRASIL, 2007). Contudo, não foi possível identificar, através dos dados estudados, o motivo do abandono do tratamento.

\section{CONCLUSÃO}

A hanseníase está relacionada às condições sociais, nas quais se destacam fatores socioeconômicos, acesso a um serviço de saúde qualificado e estigma acerca da doença. Partindo desse pressuposto, o enfermeiro tem um papel muito importante, pois o mesmo acompanha o paciente desde a procura por atendimento até a cura ou abandono do tratamento, com isso, a abordagem da enfermagem deve ser a mais acolhedora possível, exercendo os fundamentos do olhar holístico e da humanização.

O estudo permitiu identificar o sexo mais acometido, o qual foi definido como o masculino, faixa etária dos casos de hanseníase em indígenas de 15 a 19 anos, forma clínica mais prevalente e o ano de maior prevalência dos casos notificados.

Portanto, a partir deste estudo, observou-se que a atenção à saúde dos povos indígenas deve ser diferenciada e com isso ressalta-se a importância da realização de novos estudos que busquem aprofundar as discussões sobre o tema da hanseníase na população indígena.

\section{REFERÊNCIAS}

ARAÚJO, Marcelo Grossi. Hanseníase no Brasil. Rev. Soc. Bras. Med. Trop. Uberaba, v. 36, n. 3, 2003.

BRASIL. Ministério da Saúde. Secretaria de Políticas de Saúde. Departamento de Saúde, Manual Atenção Básica. Guia para o controle da hanseníase. Brasília: Ministério 2002. Série Cadernos de Atenção Básica; n. Io) - (Série A. Normas e manuais técnicos; n. III). Disponível em: http://bvsms.saude.gov.br/bvs/publicacoes/guia_de_hanseniase.pdf> Acesso 30 Ago. 2021.

BRASIL, Ministério da Saúde. Programa Nacional de Controle da Hanseníase, 2007. 
BRASIL. Ministério da Saúde. Secretaria de Vigilância em Saúde. Departamento de Vigilância das Doenças Transmissíveis. Guia prático sobre a hanseníase. Ministério da Saúde, 2017.

BRASIL, Ministério da Saúde. Secretaria de Vigilância em Saúde. Departamento de Vigilância Epidemiológica. Guia de vigilância em saúde: volume único, 3.ed., 2019

BRASIL. Ministério da Saúde. Secretaria de Vigilância em Saúde. Departamento de Doenças de Condições Crônicas e Infecções Sexualmente Transmissíveis. Boletim Epidemiológico Especial: Hanseníase. Brasília: Ministério da Saúde, 2020.

CARVAlHO, Keila Auxiliadora. Colônia Santa Izabel: a lepra e o isolamento em Minas Gerais (1920-1960). Tese de Doutorado em História. Universidade Federal Fluminense, Niterói, 2012.

\section{DINIZ, R F SITUAÇÃO EPIDEMIOLÓGICA DA HANSENÍASE NO MUNICÍPIO DE JERICÓ-PB, NO PERÍODO DE 2001 A 2012. Trabalho de Conclusão de Curso} (Graduação em Enfermagem) - Universidade Federal de Campina Grande. Cajazeiras-PB, 2013.

GUSSO. Gustavo; LOPES, José Mauro Ceratti. Tratado de Medicina de Família e Comunidade: Princípios, Formação e Prática. Artes Médicas, 2018.

Instituto Brasileiro de Geografia e Estatística (IBGE). Indicadores Sociodemográficos de Saúde no Brasil, Rio de Janeiro; 2010.

LIMEIRA OM, Gomes CM, Morais OO, Cesetti MV, Alvarez RRA. Active search for leprosy cases in Midwestern Brazil: a serological evaluation of asymptomatic household contacts before and after prophylaxis with bacillus Calmette-Guérin. RevInstMed Trop. 2013;55(3):173-7

Lobo JR, Barreto JCC, Alves LL, Crispim LC, Barreto LA, Duncan LR, et al. Perfil epidemiológico dos pacientes diagnosticados com hanseníase através de exame de contato no município de Campos dos Goytacazes, RJ. Rev Bras Clin Med. 2011; 9(4): 283-7. 
MARTINS, Marcos Antônio. Qualidade de vida em portadores de hanseníase. Dissertação de Mestrado em Psicologia- Universidade Católica Dom Bosco. Mato Grosso do Sul: Campo Grande, 2009.

Monot, M, Honore N, Garnier T, Araoz R, Coppe J, Lacroix C, et al. On the Origin of Leprosy. Science. 2005; 308: p. 1040-1042

OLIVEIRA JCF, et al. Análise do perfil epidemiológico da hanseníase em Maricá, Rio de Janeiro: uma contribuição da enfermagem. Revista de Enfermagem UERJ, 2014

Saito MI, Silva LEV. Adolescência: prevenção e risco. Rio de Janeiro (RJ): Atheneu; $200 I$.

SANTOS ES. Aspectos geográficos e epidemiológicos da hanseníase em Cuiabá e Várzea Grande-MT [tese de doutorado]. São Paulo: Faculdade de Filosofia, Letras e Ciências Humanas da Universidade de São Paulo; 2012.

SILVA, Leicy Francisca da. História da lepra ou da hanseníase? O problema da terminologia na história da doença. Anais do XXVI Simpósio Nacional de História. São Paulo, julho 20II.

TEOFILO, Da Silva. Hanseníase em indígenas no Brasil no período de 2001 a 201r. Tese (Mestrado em Saúde Coletiva) - Universidade Federal de Mato Grosso, Cuiabá, 2014.

TERRA, Fernando. Esboço histórico da lepra no Brasil. Anais Brasileiros de Dermatologia, Rio de Janeiro, v.I, n.6, p. III-VII, 1995. 